\title{
Towards free 3D end-point control for robotic-assisted human reaching using binocular eye tracking
}

\author{
Roni O. Maimon-Dror ${ }^{*, 1,2}$, Jorge Fernandez-Quesada*,1, Giuseppe A. Zito ${ }^{1}$, \\ Charalambos Konnaris ${ }^{1}$, Sabine Dziemian ${ }^{1}$, and A. Aldo Faisal ${ }^{1,2,3,4}$, Member IEEE
}

\begin{abstract}
Eye-movements are the only directly observable behavioural signals that are highly correlated with actions at the task level, and proactive of body movements and thus reflect action intentions. Moreover, eye movements are preserved in many movement disorders leading to paralysis (or amputees) from stroke, spinal cord injury, Parkinson's disease, multiple sclerosis, and muscular dystrophy among others. Despite this benefit, eye tracking is not widely used as control interface for robotic interfaces in movement impaired patients due to poor human-robot interfaces. We demonstrate here how combining 3D gaze tracking using our GT3D binocular eye tracker with custom designed 3D head tracking system and calibration method enables continuous 3D end-point control of a robotic arm support system. The users can move their own hand to any location of the workspace by simple looking at the target and winking once. This purely eye tracking based system enables the end-user to retain free head movement and yet achieves high spatial end point accuracy in the order of $6 \mathrm{~cm}$ RMSE error in each dimension and standard deviation of $4 \mathrm{~cm}$. 3D calibration is achieved by moving the robot along a 3 dimensional space filling Peano curve while the user is tracking it with their eyes. This results in a fully automated calibration procedure that yields several thousand calibration points versus standard approaches using a dozen points, resulting in beyond state-ofthe-art 3D accuracy and precision.
\end{abstract}

\section{INTRODUCTION}

Brain-Machine Interfaces (BMIs) are the most direct interface linking a user's mental intention to act via a robotic system that acts out their intention for them. BMIs are used by patients with various forms of movement impairments, be it paralysis e.g. from spinal-chord injury or stroke, lack of limbs e.g. for amputees or patients with developmental disorders or other degenerative diseases that impair their ability to move. Different Brain-Machine Interface approaches can be categorised as non-invasive cortical interfaces (e.g. EEG), invasive cortical interfaces, e.g. implanted multi-electrode arrays (MEA), or non-invasive and non-cortical interfaces (e.g. EMG). The clinical aim, remains the same: to extract an intention signal from a patient, for which conventional approaches such as joystick, mouse movement or sip and puff control are not possible. Current approaches however come at considerable clinical and post-clinical cost [1], while posing limitations for use in daily applications due to low information transmission bandwidths. Powered continuous wheelchair control requires about $15.3 \mathrm{bit} / \mathrm{s}$ and full-finger hand prosthetics would require $54.2 \mathrm{bit} / \mathrm{s}$, well beyond the

Brain \& Behaviour Lab - ${ }^{1}$ Dept. of Bioengineering \& ${ }^{2}$ Dept. of Computing, Imperial College London, South Kensington Campus, SW7 2AZ, London, UK, ${ }^{3}$ Data Science Institute, ${ }^{4} \mathrm{MRC}$ London Institute of Medical Sciences. ${ }^{*}$ joint first authors.

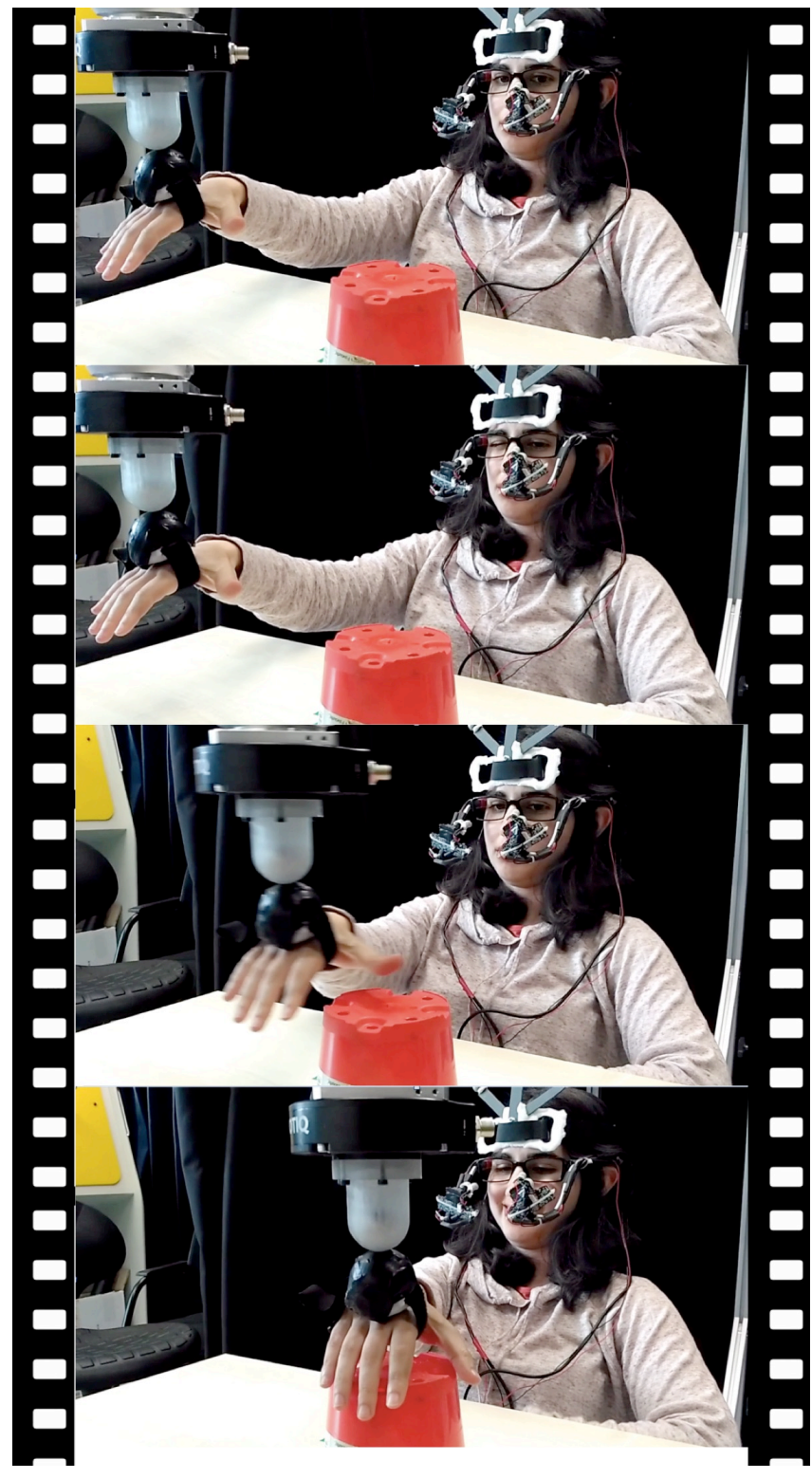

Fig. 1. Video snapshots of the system in operation. (1) The user looks at a target (red cylinder) while the 3D location of his gaze is continuously monitored by our GT3D eye tracker. (2) The user's winks trigger the robot actuator to start moving (3) and, finally (4) stopping at the 3D location of the triggering moment's gaze-target over the red cylinder enabling the user to grasp it.

reported performance of current BMI approaches (EEG 1.63 bit/s, cortical multielectrode arrays (MEA) - $3.3 \mathrm{bit} / \mathrm{s}$, noncortical non-invasive BMIs, e.g. EMG - 2.66 bit/s [2]). These BMI approaches come at significant clinical and equip- 
ment cost in the region of 25,000 USD for EEG, 150,000 USD for MEA and 15,000 USD for EMG [3] without training/rehabilitation costs. We have recently proposed a non-invasive and ultra-low cost alternative BMI approach, the GT3D system, based on intention decoding from 3D gaze signals. This enables real-time closed-loop control that outperforms invasive (and non-invasive) BMIs in terms of cost and read-out data rates [3] and hence enables robotic arm control in conjunction with other low data-rate signal sources (EMG [4], EEG [5] or tongue-flick-switches [6]. As we have previously estimated, our GT3D approach could yield bit rates up to $43 \mathrm{bit} / \mathrm{s}$ at a prototype cost of $<30$ USD and we have framed this performance within a comparison of BMI approaches in terms of cost and information throughput [3]. Although eye tracking and even low-cost eye tracking is nothing new, we showed that gaze location, particularly in 3D and at high-data rates (matching those of eye movements) provides a real-time decodable and graded control signal that should be utilised in the BMI field. Eye movements, retained by patients with serious motor deficiencies, paralysis and limb amputation [7] the only directly observable behavioural signals that are naturally both highly correlated with actions at the task level, and proactive of our peripheral motor actions [8], [9]. Having addressed cost, information throughput, latency and minimising invasiveness, an additional barrier for current BMIs is the extensive preparation, set up and patient learning time, which can reach months or even years. This poses a major challenge for patient uptake of BMI based control of robotic devices [10].

Consequently we propose [3] how tracking the gaze position in 3D using our custom developed binocular 3D eye trackers (GT3D), provides a considerably richer signal directly relevant for robotic, exoskeleton and prosthetic control, overcoming the limitations of conventional BMI systems. We have since shown that such eye tracking can be used for precise robotic end-point control and manipulation in small 3D volumes [11] and for near instant ability to perform teleoperation tasks as painting in an oil brush technique [12], or navigate a wheelchair without requiring to interact with a user interface [13]. Here we demonstrate a novel technique for $3 \mathrm{D}$ calibration that enables us to quickly and accurately calibrate a UR-10 robotic arm in 3D space. The arm endpoint provides a novel magnetic safety gimbal that allows us to safely link a users hand or wrist to the arm so as to act as arm support system. This arm is controlled by the 3D gaze point and triggered to move to the present location of $3 \mathrm{D}$ gaze using a simple wink command.

\section{METHODS}

Our objective is to design and implement a functional three-dimensional eye-gaze driven robotic arm system that moves a paralysed hand to a desired target. A number of commercial and research-originated eye and head tracking systems currently exist.

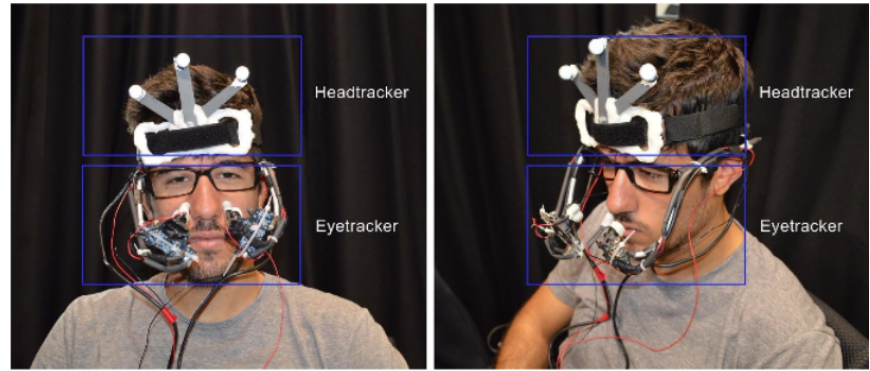

Fig. 2. Photographs of GT3D eye tracker with head band IR marker "crown" for optical head tracking. (Left) Front and (Right) side view.

\section{A. System components: Hardware}

In order to create a suitable system, three main components must be included in the system design: a reliable robotic arm, a high precision eye tracking device and an accurate motion tracking solution i.e a head tracker. A fundamental system design requirement is versatility. Hence, the system must be easily updatable in the future by improved hardware components like other types of robotic actuators, eye tracking devices and motion tracking solutions.

a) UR-10 robotic arm: The chosen actuator for the system is the industrial robotic arm UR10 by Universal Robots. This robotic arm features 6 degrees of freedom and can hold a maximum payload of $10 \mathrm{~kg}$ with a reach radius of $1.3 \mathrm{~m} \mathrm{[14].} \mathrm{Within} \mathrm{our} \mathrm{system} \mathrm{the} \mathrm{UR10} \mathrm{is} \mathrm{controlled}$ through a custom-made application integrated in the ROS environment on a Linux computer. For user safety reasons the robot has been constrained in its motions using security planes and preloaded models of the workspace that the robot will never enter with any part [12]. In addition, at all times the system's operator holds an emergency stop button to be able to immediately stop motion execution [15].

b) GT3D Eyetracker: Eye tracking devices are the input devices that record and send eye gaze information to the rest of the system. In this project we decided to use a mobile, wearable eye tracker, as it provides the versatility required for this kind of application and allows us to compensate for head movements when a head tracker is attached to it. 3D eye trackers have to be binocular head-mounted camerabased systems that monitor the rotations of both eye balls so as to determine from vergence the direction and distance of the gaze target. We use our GT3D binocular eye tracker: a custom-made, low-cost and portable eye tracking device [3]. The device is built from two modified commercial PlayStation 3 cameras attached to an aluminium and plastic frame. The position of the cameras is adjustable to suit the user's eyes location. In order to record the user's gaze features, there are two IR LEDs attached to the cameras that increase pupil reflection and ease the acquisition of user's pupil images at a maximum frequency of up to $150 \mathrm{~Hz}$ and a resolution of $320 \times 240$ pixels. Further post-processing is carried out to filter and binarize recorded images to extract the pupil center coordinates, which is used later as the input signal to calibrate and control the system. Due to the customizable nature of the device, it is possible to modify the zoom area, region of interest within the image, set a 
contrast threshold for the binarised image and apply ellipse fitting parameters during system operation to optimise the performance according to the user's particular features. The GT3D eye tracker is connected to a Windows computer via 3 USB ports: one for each of the cameras and one to light the IR LEDs.

c) Headtracking: One of the main challenges of mobile eye tracking is that current calibration and control algorithms are very sensitive to head movements. To account for these shifts, the user wears a head tracking markers in form of a "crown". These markers are tracked by an OptiTrek Flex13 system (Natrual Point Inc, Corvallis, OR) with 3 cameras that feeds our system with information regarding translational and orientational head movements during robot control mode. Head tracking is anabled by three reflective markers placed onto a PVC and polymorph rigid body "crown" that the user will strap around their head while using the system. Once the head tracker is placed, a rigid body is defined from the fixed conformation of the reflective markets. Markers were placed in an asymmetrical conformation to provide a clear distinction of orientation and translation within the rigid body and thus movements of the head (see Fig. 2).

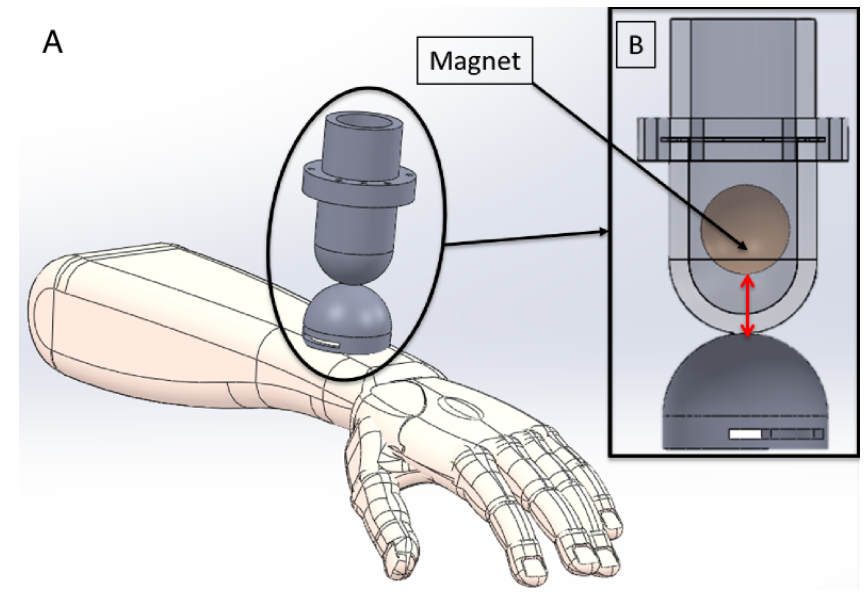

Fig. 3. Design of bi-hemispherical magnetic safety gimbal. (A) One hemisphere belongs to the wrist attachment point with slits at its base for fabric hook and loop fastener straps to pass through and around wrist or hand. The other hemisphere is mounted at the end of a (B) hollow cylindrical tube attached to the robot end-point with ball magnet insertion.

d) Hand/Arm safe attachment and gimbal: We developed a 3D printed attachment system (see Fig. 3) based on a magnetic wrist band worn by the user and a magnetic pylon attached to the UR-10 endpoint. Both the pylon and the wrist band have hemispherical end-points in which two spherical magnets were inserted. This attachment system enables the user's wrist to rotate freely with 3 degrees of freedom while being translated in 3D dimensions by the robot, thus acting as a gimbal. Magnet strength and separation was chosen so as to acts a safety feature should robot translation exceed safety critical forces. Moreover, if the two hemispherical attachment endpoints rotate away from each other's zenit by over $90^{\circ}$, then the increasing separation of the two magnetic balls would lower the magnetic forces to the point that the mass of the arm would automatically detach the wrist from the robot. The gimbal can be mounted with fabric hook and loop fastener straps either around the wrist or around the palm of the hand.

\section{B. System components: Software architecture}

To meet the requirement of a versatile homan-robot interactive system, the software must use a standardised communication to facilitate easy combination or subsitution of hardware components and integration of further humanmachine interfaces and actuators. We have therefore taken a software engineering approach and have adopted a common middleware to connect the various system components.

a) ROS as middleware: The Robot Operating System (ROS) is an open-source meta-operating system supported by the Open Source Robotics Foundation (OSRF) which acts as our system's middle ware. ROS is particularly suitable for distributed applications like our system as it provides standardised solutions for synchronous and asynchronous communication between processes that run on multiple machines. ROS includes operating system functionalities that are beyond the scope of a regular operating system, package management and hardware abstraction that maintains direct access to low-level hardware resources are such operating system functionalities. Robotic actuators and sensors come either with preexisting ROS drivers (UR-10) or have been implemented by ourselves (eye tracker, head tracker).

b) Software Architecture: Our software architecture (see Fig. 4) includes the following main components: an eye tracker data streamer, a head tracker data streamer, a robot position streamer, a robot controller and a manager that is responsible for calibration, control, and communicating with all other components. The connection between the manager and the eye and head trackers is done via a simple protocol over TCP-IP. This allows to easily exchange eye trackers by writing a simple converter between the specific eye tracker streaming protocol and the systems protocol. The interaction with the robot is done by implementing a basic ROS-service called move_robot with an input of a 3D end-point. The robot also sends a continuous stream of ROS-messages reporting its current end-effector position. The manager steers the streams between the calibration and control modes and uses the service to control the robot thus creating complete decoupling between the different parts of the system. The components are written in Python and $\mathrm{C}++$. Trajectory planning and execution is done using the ROS move-it package. The manager, robot (UR10) controller and position streamer are written as ROS-nodes.

c) Calibration: There are only few approaches for 3D calibration of eye trackers (reviewed in [3]), and these typically rely on the user need to have to look at a discrete point in a fixed position of space to generate a calibration model, and occasionally involving the experimenter having to move the calibration visual display unit forward and backward. In contrast our system makes use of the fact that we have a robot arm in the system. This enables a dynamic calibration procedure to model the relationship between the eye features and the 3D gazed location. We first demonstrated[11] this 


\section{Eye-gaze driven robotic arm system overview}

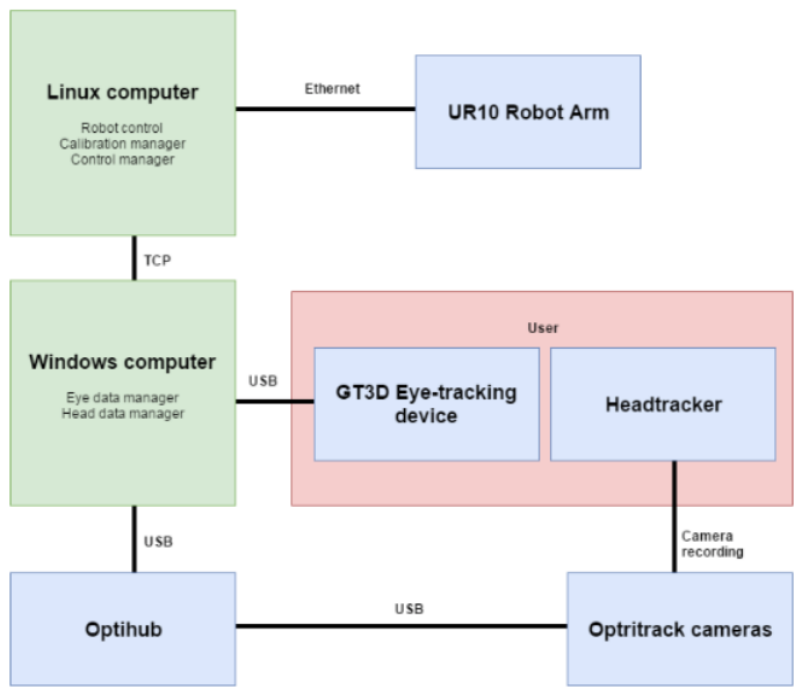

Fig. 4. System architecture of the gaze-controlled robot arm. Blue boxes are hardware components, green boxes computers. The pink box indicates the user and comprises worn hardware. Black lines designate data flow and data link type.

method for a head-fixed setup and have now generalised it to enable full, free head movements. In our procedure, the robot moves in a continuous 3D trajectory that spans the working volume following a space-filling path while the user tracks a the robot's end-effector during the two minute motion. The three data streams (end-effector position, eye and head tracking) are synchronised and resampled to a comment rate and fed into the Gaussian Process (GP) Regression based calibration algorithm[11]. This algorithm treats the collection of $n$ inputs as a multivariate normal distribution ( $n$-dimensional) and the resulting fitting process produces the joint probability distribution of all input random variables. In this case a GP model was fitted for each of the 3 axes of the robot's movement $(x, y, z)$ resulting in 3 models which receive 4 eye features ( $x, y$ coordinates of the left and right pupil) as input and return a mean and standard deviation representing the predicted location in a single axis. Since the quality of calibration depends on the ability of the subject to follow continuously throughout the calibration procedure, the robot performs a second, test trajectory to assess the quality of calibration empirically and if necessary repeat it. 100 data points from the test trajectory is fed to the model and the mean error between the predicted position and the actual robot position is calculated. This information is presented to the experimenter to determine whether the calibration procedure should be repeated. During the calibration procedure the head should be kept relatively still as the head position data is not a part of the GP model. The position of the head obtained from the head tracker during calibration is saved as the reference position and all following predictions will be shifted and rotated in relation to the difference between the current head position from the reference. The head position data was not integrated in to the GP model for two reasons, first the GP model is most useful for noisy data, which is not the case for our head
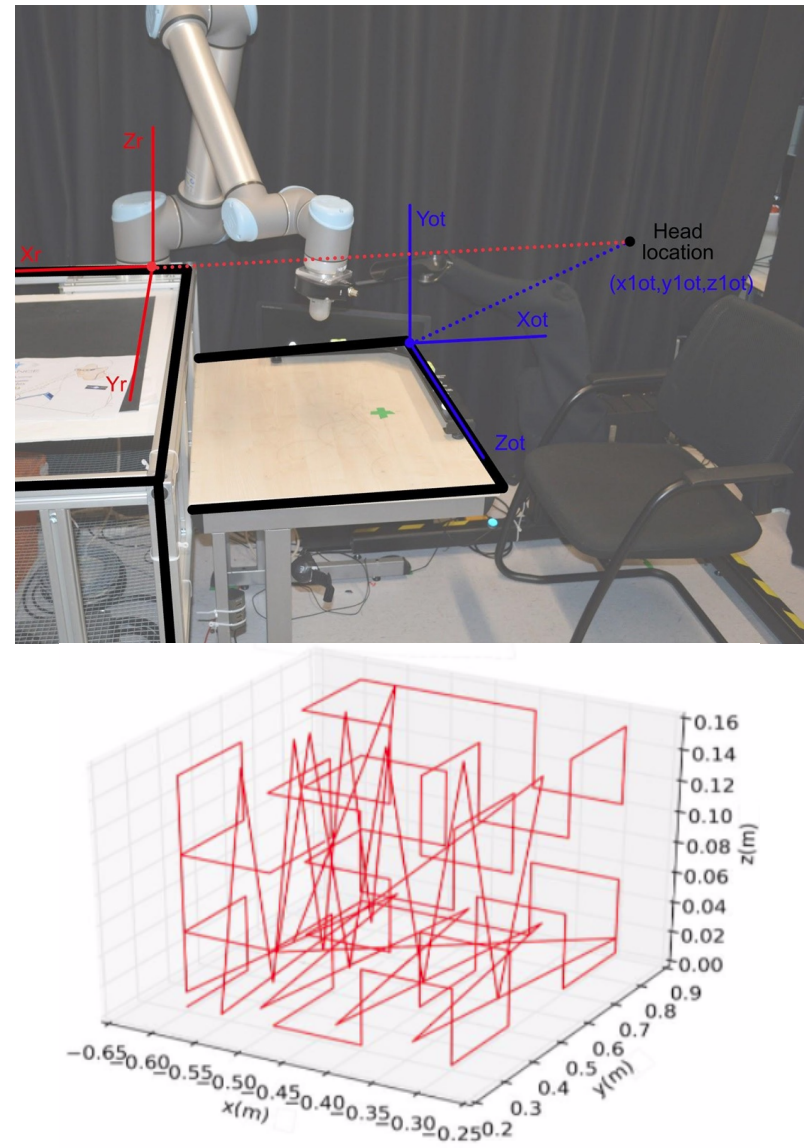

Fig. 5. (Top) Photograph of the setup with corresponding coordinate systems. (Bottom) Calibration trajectory of the robot arm end-point tracked by users.

position stream, and where the mapping from input to output is unclear. In this case, the GP model maps pupil position data to a gaze vector which is then rotated and translated according to the head orientation and position. Second, in order to create a reliable model for both head and eye data one would need the calibration procedure to span across head positions and rotations as well as pupil positions, resulting in a much longer calibration process, as more data points are required due to the doubling in the number of input dimensions.

d) Control: After performing the calibration procedure and computing the GP model to control the robotic arm, the user is able to operate the robotic arm straightaway. The system provides full control of the robotic arm within the working volume by means of voluntary winks. When the user wants to move the robot to a certain location (e.g. place the robotic arm onto an object), this motion is accomplished by winking any of the eyes for a preset length of time. In order to produce optimal performance, a median gaze point of the second prior to winking is taken as the intended location to avoid outliers reported by the eye tracker during the wink.

e) System setup: The setup design is chosen such that it facilitates user interaction with the whole system and additionally keeps the user safe at all times. The UR10 robotic arm is attached to a metallic framework in front of the 
user. Adjacent to the frame, there is a desk of the dimensions $60 \mathrm{~cm} \times 70 \mathrm{~cm}$. This desk is also used as a reference surface to define several elements of the workspace. It is used to set up the ground plane of the OptiTrack coordinate system and define the home point of the robot application. Further it serves to place target goals on it used to validate the overall performance of the system. When operating the system the user is sitting on a chair in front of the desk. Thereby, the user is in immediate reach and has a clear view of the robotic arm and the complete workspace.

f) Performance evaluation: To assess the performance of the system, subjects are asked to look at an object that is positioned at 11 different points across two semi-circular arches $(13 \mathrm{~cm}$ and $26 \mathrm{~cm}$ in radius, respectively) spanning the desk surface. For each of the locations, subjects are asked to perform the selection command (i.e. wink for one second) while staring at the object. This procedure is performed five times to assess the repeatability of the system in control mode. Acoustic feedback is produced every time the user produced a selection command.

\section{RESUlTS}

We evaluated the system with $N=5$ healthy subjects (aged 23-35). All subjects were able to perform end-point control of the robot using eye wink commands after fixating on a location in their three dimensional work space and then to move the robot to that position. In situations where the robot did not accurately enough reach the desired end-point they were able to reposition the robot with a corrective movement so that the target object was within their grasp. Table I lists the mean absolute error obtained in the usability experiment for each subject along three dimensions and the Euclidean error. From the results, we can observe that the highest error belongs to the depth dimension (i.e. $7.7 \mathrm{~cm}$ ). This outcome was expected as pupil coordinates change less dramatically when observing an object moving along that direction. Indeed, overcoming this issue is the main challenge of 3D eye tracking techniques. Standard variations around the mean of the end-point are also higher along the depth axis, due to the higher variability associated to predictions along this axis.

When classifying the results in reference to the point where the user is looking at, results show that the system performs slightly better in the inner area, whereas mean absolute errors and standard deviations increase for the outer area of the system. In all cases the users were able to move their hand (attached to the robot) towards the targets. The robot's stiffness itself prevented any voluntary or involuntary arm movements biasing the end-point of the robot.

\begin{tabular}{|l|c|c|}
\hline & Mean absolute error $(\mathrm{cm})$ & Standard dev $(\mathrm{cm})$ \\
\hline Depth axis [X] & 7.7 & 6.6 \\
\hline Horizontal axis [Y] & 4.0 & 2.4 \\
\hline Vertical axis [Z] & 6.2 & 2.7 \\
\hline Euclidean & 12.0 & 4.1 \\
\hline
\end{tabular}

TABLE I

SUMMARY OF ERRORS IN USABILITY EXPERIMENT FOR $N=5$ SUBJECTS

\section{Discussion}

Our system demonstrates continuous gaze-based end-point control using a hand-arm support system, capable of moving a paralysed users hand and arm to a desired end-point. The integration of the system was successful, and the developed setup allowed for eye-gaze based three-dimensional control of the robotic arm in an environment similar to the one that target users will encounter in desk-based or wheelchairtablet based settings. As from the results obtained during control mode, the standard deviation of the end-point from the target averages $4.1 \mathrm{~cm}$ for the eleven locations set for the usability experiment. We report this variability across all 3 dimensions, as well as an error per dimension, however other real-time closed loop operated EEG or EMG systems, which either actuate a tele-operated robotic systems or wearable exoskeletons, typically do not report intepretable end-point errors and mostly report indirect measures such as success rate in reaching a target [16], [17], [18], [19]. However, it is difficult to estimate the accuracy of movement endpoint as target size/shape and/or the modality with which a target needs to successfully reached, hovered over or grasped are left unclear. Moreover, most of these robotic systems enable the user to continuously adjust the trajectory of the arm and correct for movement error - in contrast our system operates ballistically, in that, once a target location is selected by looking at it, the arm will move there (we did not allow for corrective movements to be included in our trials). In one case we were able to evaluate the end-point error based on results for invasive cortical Brain-MachineInterfaces controlling a robotic arm with the same degrees of freedom as ours: We can convert the reported probability of touching fixed targets of known sizes [20], [21] to an estimate of the standard deviation of the end-point error which results in $4.6 \mathrm{~cm}$ of end-point variability they must achieve, which is somewhat higher than to our $4.1 \mathrm{~cm}$. In both cases the variability of the end-point is smaller than the open hand of the user, meaning that objects could we always grasped.

In contrast to our previous paper [11], we present here the results of several qualitative improvements: 1. we advanced from a head-fixed setup to a free head movement setup. 2 . we moved from a small robot arm with a workspace adjacent to the user's face, to a large robot which operates in the workspace of a free moving human arm and operated at "arm's length" away from the users. 3. we introduce a novel magnetic safety gimbal that enables us to comfortably attach the hand of the user to a robot arm end-effector. In this study, we focus on the practical usability of the system, which is why we report the performance of the system in real time control in order to reach a specific end point specified by the user in the task, in contrast to the calibration errors reported in the previous paper. Here, we utilise a fully automated calibration procedure that yields several thousand calibration points versus standard approaches using a dozen points on planes of various depths - which is a novel approach not requiring human assistance for the user in calibrating the system, resulting in beyond state-of-the-art 3D accuracy and 
precision for continuous controlled robotic actuators. Winkbased triggering of movement is an attractive solution given that binocular eye data is already available, and is objectively measured more reliable than other modalities such as voicebased or EMG-based triggering [22].

We believe that further improvements in the head tracker design, by integrating it in the eye tracker to form one rigid body, will enable us to obtain more accurate results and better usability of the system. The current head-tracking setup using optical motion tracking which requires a camera and markers require some form of rigid arrangement of the camera with respect to the robotic arm by having a static relationship between camera and robot arm, such as here using a dedicated work space or on a robot-mounted wheelchair. IMU (inertial measurement units) based tracking could substitute optical tracking of head orientation, such as in a head-mouse [23], but is limited by the poor ability of IMUs to track translation of the head accuratley over time. Thus optical, radar, magnetic or mechanical tracking of head location is currently necessary, unless the end-user is constrained by their disease or their head rest on a wheel-chair in their head movements. In conclusion, most examples in the literature accomplish three-dimensional control of robotic actuators by means of hybrid interfaces that combine eye-tracking for discrete target selection with other signal sources, such as depth sensors and computer vision [24], brain-machine interfaces [20], gestures [12] and speech commands [25]. We demonstrate an assistive robotic arm attached to the user which is solely guided by eye commands. Fhis proof of concept represents a promising application of eye-tracking technology.

Acknowledgements: This research was supported by eNHANCE (http://www.enhance-motion.eu) under European Union's Horizon 2020 research and innovation programme grant agreement No 644000.

\section{REFERENCES}

[1] J. J. Shih, D. J. Krusienski, and J. R. Wolpaw, "Brain-computer interfaces in medicine," in Mayo Clinic Proceedings, vol. 87, no. 3. Elsevier, 2012, pp. 268-279.

[2] O. Tonet, M. Marinelli, L. Citi, P. M. Rossini, L. Rossini, G. Megali, and P. Dario, "Defining brain-machine interface applications by matching interface performance with device requirements," Journal of Neuroscience Methods, vol. 167, no. 1, pp. 91 - 104, 2008.

[3] W. W. Abbott and A. A. Faisal, "Ultra-low-cost 3d gaze estimation: an intuitive high information throughput compliment to direct brainmachine interfaces," Journal of neural engineering, vol. 9, no. 4, p. 046016, 2012.

[4] E. Corbett, K. Kording, and E. Perreault, "Real-time evaluation of a noninvasive neuroprosthetic interface for control of reach," IEEE Transactions on Neural Systems and Rehabilitation Engineering, vol. 21, no. 4, pp. 674-683, 2013.

[5] G. Onose, C. Grozea, A. Anghelescu, C. Daia, C. J. Sinescu, A. V. Ciurea, T. Spircu, A. Mirea, I. Andone, A. Spanu, C. Popescu, A.-S. Mihaescu, S. Fazli, M. Danoczy, and F. Popescu, "On the feasibility of using motor imagery eeg-based brain-computer interface in chronic tetraplegics for assistive robotic arm control: a clinical test and longterm post-trial follow-up," Spinal Cord, vol. 50, no. 8, pp. 599-608, Aug 2012.

[6] M. Buckley, R. Vaidyanathan, and W. Mayol-Cuevas, "Sensor suites for assistive arm prosthetics," in Computer-Based Medical Systems (CBMS), 2011 24th International Symposium on. IEEE, 2011, pp. $1-6$.
[7] H. J. Kaminski, C. R. Richmonds, L. L. Kusner, and H. Mitsumoto, "Differential susceptibility of the ocular motor system to disease," Annals of the New York Academy of Sciences, vol. 956, no. 1, pp. 42-54, 2002.

[8] D. H. Ballard and C. M. Brown, "Principles of animate vision," CVGIP: Image Understanding, vol. 56, no. 1, pp. 3-21, 1992.

[9] M. F. Land, D. N. Lee, et al., "Where we look when we steer," Nature, vol. 369, no. 6483, pp. 742-744, 1994.

[10] T. R. Makin, F. de Vignemont, and A. A. Faisal, "Neurocognitive barriers to the embodiment of technology," Nature Biomedical Engineering, vol. 1, p. 0014, 2017.

[11] P. M. Tostado, W. W. Abbott, and A. A. Faisal, "3d gaze cursor: Continuous calibration and end-point grasp control of robotic actuators," in Robotics and Automation (ICRA), 2016 IEEE International Conference on. IEEE, 2016, pp. 3295-3300.

[12] S. Dziemian, W. W. Abbott, and A. A. Faisal, "Gaze-based teleprosthetic enables intuitive continuous control of complex robot arm use: Writing \& drawing," in Biomedical Robotics and Biomechatronics (BioRob), 2016 6th IEEE International Conference on. IEEE, 2016, pp. 1277-1282.

[13] S. I. Ktena, W. Abbott, and A. A. Faisal, "A virtual reality platform for safe evaluation and training of natural gaze-based wheelchair driving," in Neural Engineering (NER), 2015 7th International IEEE/EMBS Conference on. IEEE, 2015, pp. 236-239.

[14] Universal Robots A/S, "Technical specifications UR10, Item no. 110110," Universal Robots A/S, Energivej 25, DK-5260 Odense S, Denmark, Tech. Rep., 2014.

[15] J. F. Quesada, "Design and Integration of an Eye-Gaze Driven Robotic Arm System: Business Plan and Research Thesis," Master's thesis, Imperial College London, 2016.

[16] P. K. Artemiadis and K. J. Kyriakopoulos, "Emg-based control of a robot arm using low-dimensional embeddings," IEEE Transactions on Robotics, vol. 26, no. 2, pp. 393-398, 2010.

[17] J. Meng, S. Zhang, A. Bekyo, J. Olsoe, B. Baxter, and B. He, "Noninvasive electroencephalogram based control of a robotic arm for reach and grasp tasks," Scientific Reports, vol. 6, 2016.

[18] D. Blana, T. Kyriacou, J. M. Lambrecht, and E. K. Chadwick, "Feasibility of using combined emg and kinematic signals for prosthesis control: A simulation study using a virtual reality environment," Journal of Electromyography and Kinesiology, vol. 29, pp. 21-27, 2016.

[19] J. Lobo-Prat, P. N. Kooren, M. M. Janssen, A. Q. Keemink, P. H. Veltink, A. H. Stienen, and B. F. Koopman, "Implementation of emg-and force-based control interfaces in active elbow supports for men with duchenne muscular dystrophy: a feasibility study," IEEE transactions on neural systems and rehabilitation engineering, vol. 24, no. 11, pp. 1179-1190, 2016.

[20] D. P. McMullen, G. Hotson, K. D. Katyal, B. A. Wester, M. S. Fifer, T. G. McGee, A. Harris, M. S. Johannes, R. J. Vogelstein, A. D. Ravitz, W. S. Anderson, N. V. Thakor, and N. E. Crone, "Demonstration of a semi-autonomous hybrid brain-machine interface using human intracranial eeg, eye tracking, and computer vision to control a robotic upper limb prosthetic," IEEE Transactions on Neural Systems and Rehabilitation Engineering, vol. 22, no. 4, pp. 784-796, 2014.

[21] J. L. Collinger, B. Wodlinger, J. E. Downey, W. Wang, E. C. TylerKabara, D. J. Weber, A. J. McMorland, M. Velliste, M. L. Boninger, and A. B. Schwartz, "High-performance neuroprosthetic control by an individual with tetraplegia," The Lancet, vol. 381, no. 9866, pp. 557-564, 2013.

[22] B. Noronha, S. Dziemian, G. Zito, C. Konnaris, and A. Faisal, ""wink to grasp" - comparing eye, voice \& emg gesture control of grasp with soft-robotic gloves," in Rehabilitation Robotics (ICORR), 2017 IEEE 15th Intl. Conference on, vol. 15. IEEE, 2017, pp. 7-12.

[23] N. Sim, C. Gavriel, W. W. Abbott, and A. A. Faisal, "The head mouse - head gaze estimation "in-the-wild" with low-cost inertial sensors for bmi use," in Neural Engineering (NER), 2013 6th International IEEE/EMBS Conference on. IEEE, 2013, pp. 735-738.

[24] M. Leroux, S. Achiche, and M. Raison, "Assessment of accuracy for target detection in 3d-space using eye tracking and computer vision," PeerJ Preprints, vol. 5, p. e2718v1, 2017.

[25] D. Rozado, L. Stephen, and N. Kottege, "Interacting with objects in the environment using gaze tracking glasses and speech," in Proceedings of the 26th Australian Computer-Human Interaction Conference on Designing Futures: the Future of Design. ACM, 2014, pp. 414-417. 